\title{
Estudio del desempeño y liderazgo de estudiantes de ingeniería industrial usando análisis de redes sociales
}

\author{
Study of performance and leadership in industrial \\ engineering students using social network analysis \\ Giuliani Coluccio Piñones ${ }^{1 *} \quad$ Maritza Muñoz-Pareja ${ }^{2}$ \\ Recibido 1 de diciembre de 2020, aceptado 30 de septiembre de 2021 \\ Received: December 1, 2020 Accepted: September 30, 2021
}

\begin{abstract}
RESUMEN
En este estudio se analizó la relación entre el liderazgo y el desempeño en equipos de estudiantes de educación superior. Para esto, primero, se conceptualizó el liderazgo como un proceso de influencia entre los miembros del equipo y, en segundo lugar, se modeló este proceso del equipo usando análisis de redes sociales. Para estudiar la relación propuesta entre liderazgo y desempeño, se llevó a cabo un estudio a 164 estudiantes en 41 equipos estudiantes de Ingeniería Civil Industrial en dos universidades de Chile. Usando modelos de regresiones lineales, nuestros resultados muestran que la densidad de liderazgo de la red se relaciona positivamente con el desempeño mientras que la centralización del liderazgo en la red se relaciona de manera negativa con el desempeño de los estudiantes. Estos resultados ayudan tanto a los docentes como a las instituciones de educación superior a tener una herramienta estadística confiable con la cual puedan hacer un seguimiento sobre el desarrollo del liderazgo en los estudiantes a lo largo del plan de estudios.
\end{abstract}

Palabras clave: Liderazgo, educación superior, análisis de redes sociales.

\begin{abstract}
In this paper, we analyze the relationship between leadership and performance in teams of higher education students. For this, first, we conceptualize leadership as an influence process between team members and, second, we model this team process using social network analysis. To study the proposed relationship between leadership and performance, we conducted a study at 164 students of 41 teams of industrial engineering students of two universities in Chile. We analyzed our data using linear regression analysis, and our results showed that leadership network density is positively related to performance, and leadership network centrality is negatively related to performance. These results help teachers, and higher education institutions have a reliable statistical tool to track student leadership development throughout the curriculum.
\end{abstract}

Keywords: Leadership, higher education, social network analysis.

\footnotetext{
1 Universidad de Tarapacá. Departamento de Ingeniería Industrial y de Sistemas. Arica, Chile. E-mail: gcolucciop@uta.cl 2 Universidad Católica de la Santísima Concepción. Facultad de Medicina. Concepción, Chile. E-mail: mmunoz@ucsc.cl

* Autor de correspondencia: gcolucciop@uta.cl
} 


\section{INTRODUCCIÓN}

El liderazgo es uno de los fenómenos organizacionales que ha tenido mayor relevancia a lo largo del tiempo. En este sentido, la literatura en diferentes áreas de estudio ha buscado entender si el liderazgo puede asociarse a diferentes beneficios en los equipos [1-3]. Así, la investigación realizada se ha centrado en entender cómo las características individuales que tiene el líder formal del equipo, siendo este un puesto previamente designado, favorecen diferentes resultados positivos para el equipo [4-7]. De esta manera, se ha logrado un consenso de la importancia que tiene el liderazgo en los equipos ya que puede afectar su funcionamiento. Sin embargo, últimas investigaciones señalan que esta mirada clásica del liderazgo centrada en líder formal no captura toda la riqueza y complejidad de este fenómeno en los equipos, siendo necesario explorar nuevas conceptualizaciones del liderazgo en los equipos [8-10].

Recientes investigaciones proponen que el liderazgo es un proceso del equipo [11-14]. En este sentido, el liderazgo más que una relación formal entre el jefe y sus subordinados se entiende como un proceso donde un miembro del equipo influye en otro, formando una relación de liderazgo $[12,15,16]$. Esta conceptualización, a diferencia de la mirada centrada en el líder formal, entiende que el liderazgo es un proceso dinámico donde la influencia entre los individuos para lograr las metas genera el proceso de liderazgo en el equipo [8, 17]. Esta forma de entender el liderazgo en los equipos ha ayudado a clarificar el funcionamiento de este fenómeno por ejemplo en equipos autogestionados o que no tienen un líder formal definido. En estos equipos, al no existir una relación formal de liderazgo, se hace más probable que los diferentes miembros del equipo busquen influir en sus compañeros de equipo, favoreciendo el surgimiento del liderazgo $[12,16]$.

Dentro del estudio de equipos autogestionados y liderazgo, uno que capta especial atención es el de equipos de estudiantes $[18,19]$. Esto se debe a que las instituciones de educación superior declaran que el liderazgo es una de las principales competencias que desean formar en los estudiantes [20, 21]. Además, desde el punto de vista de las organizaciones, tener individuos que posean características de liderazgo es altamente deseable ya que estos son capaces de afrontar de manera exitosa los desafíos del mercado y tener un mejor desempeño [22]. En este sentido, se hace necesario pasar a entender no solamente las características que hacen a los estudiantes ser líderes sino también como el liderazgo del equipo se desarrolla y puede afectar el rendimiento de este $[23,24]$.

Para poder desarrollar el liderazgo en los estudiantes, las instituciones de educación superior ocupan diferentes metodologías de trabajo que fomentan la interacción y trabajo en equipo. Gran parte de estas metodologías se basan en fortalecer el aprendizaje teórico en un ambiente colaborativo donde los estudiantes traspasan información y conocimiento continuamente a sus compañeros de equipo, desarrollando diferentes características deseables en los estudiantes [18, 20, 25-27]. Sin embargo, aunque las instituciones de educación superior hacen bastantes esfuerzos por desarrollar el liderazgo en sus estudiantes, los académicos no tienen certeza si es que realmente se está desarrollando este fenómeno en los equipos de trabajo y si este último genera un mejor desempeño. Esto ya que gran parte de los estudios ha medido autopercepciones de características de liderazgo de los estudiantes más que enfocarse en el proceso de influencia entre los diferentes miembros del equipo y su relación con el desempeño [19, 28]. En este sentido, las nuevas conceptualizaciones del liderazgo colectivo pueden entregar herramientas metodológicas y estadísticas que ayuden a entender esta relación de mejor manera [29].

Uno de los principales modelos de liderazgo colectivo que ha sido capaz de conceptualizar y modelar las relaciones entre individuos en estructuras de liderazgo no formal es el liderazgo compartido [3034]. El liderazgo compartido se refiere al proceso del equipo en cual los diferentes miembros toman y ceden el liderazgo de manera dinámica dentro del equipo $[35,36]$. Este modelo de liderazgo se puede ver en el proceso formativo de los estudiantes, ya que estos al formar equipos con otros estudiantes en las diferentes asignaturas que cursan, deben cumplir con tareas altamente interconectadas que contribuyen al cumplimiento de una meta común [37]. Esta característica favorece que se formen las relaciones de liderazgo entre los estudiantes, siendo estas las bases del liderazgo compartido [31,34], [36]. Además, también se ha podido establecer que 
existe una relación consistente entre el liderazgo compartido y el desempeño de los equipos, donde estructuras más densas y descentralizadas de liderazgo se asocian a un mayor desempeño [31, $[32,34]$. Esto se debe a que en equipos donde se desarrolla más liderazgo, los miembros entregan mayores recursos para lograr las tareas, comparten información y sienten un mayor compromiso con el equipo [31], [34]. Además, cuando los miembros del equipo son capaces de aceptar y generar influencia en sus compañeros de equipo, se van formando estructuras de confianza, donde esta última tiene una estrecha relación con el desempeño de los equipos $[38,39]$. Para poder determinar estas relaciones, los investigadores han utilizado diversas técnicas estadísticas, sin embargo, la que tiene un mayor soporte metodológico es el análisis de redes sociales debido a su versatilidad en el modelamiento de estructuras en equipos utilizándose en gran parte de los trabajos realizados (por ejemplo [12, 15, 33, 40, 41]), resaltando el estudio de DeRue [35], donde expone como deben modelarse estos equipos usando el análisis de redes sociales. Este autor señala que las interacciones entre miembros del equipo deben tratarse como "ties" que van construyendo estructura de liderazgo y dependiendo de la cantidad de estos "ties", se pueden obtener diferentes configuraciones de estructuras de liderazgo en los equipos ${ }^{3}$.

Llevando esto al contexto de equipos de estudiantes, una estructura más densa de liderazgo del equipo se puede ver como todos los estudiantes van ejerciendo una cantidad de liderazgo alta en el equipo y una estructura descentralizada de liderazgo puede verse en el hecho de que todos los estudiantes ejercen el liderazgo y este no se centra en uno o unos pocos estudiantes, donde se esperaría que ambos tuviesen un efecto positivo en el desempeño. De esta manera, para poder estudiar la relación entre liderazgo compartido y el desempeño de equipos de estudiantes, se hará mediante las estructuras de liderazgo del equipo, por lo que planteamos las siguientes hipótesis de estudio:

H1. Estructuras más densas de liderazgo en equipos de estudiantes se relacionan positivamente con mayores niveles de desempeño.

\footnotetext{
3 El calculo en profundidad de estos indicadores de la red están desarrollados en la sección de medidas
}

H2. Estructuras más descentralizadas de liderazgo en equipos de estudiantes se relacionan positivamente con mayores niveles de desempeño.

\section{DISEÑO DE LOS ESTUDIOS Y PROCEDIMIENTO}

\section{Procedimiento}

Para poder responder a las hipótesis propuestas, se realizó un estudio en dos Universidades de Chile en estudiantes de la carrera de Ingeniería Civil Industrial. El primer estudio se realizó en estudiantes de la Universidad Católica de la Santísima Concepción y en segundo estudio en estudiantes de la Universidad de Tarapacá en la asignatura Modelos de Pronósticos y en la asignatura Econometría, respectivamente. Estas asignaturas en ambas universidades son asignaturas que comprenden la formación específica del Ingeniero Industrial y están ubicadas en la medianía del plan de estudios donde se fomenta el trabajo en equipo y la interacción entre los diferentes estudiantes para la realización de las tareas. En ambos estudios los alumnos en la primera clase debieron formar equipos cuatro personas con el fin de realizar un trabajo de duración de aproximadamente un mes y medio, siendo estos equipos formados de manera aleatoria. En ese instante, a los alumnos se les aplicó un cuestionario que captura información como el género, la edad y la universidad de procedencia. Luego, a las 3 semanas, que representa la mitad del trabajo, y al final del trabajo, los estudiantes tuvieron que responder una pregunta para determinar la influencia percibida por cada miembro del equipo.

\section{Medidas}

Para poder medir el liderazgo compartido en los equipos, recientes estudios han ocupado diferentes técnicas estadísticas basadas en el análisis de redes sociales (social network análisis, SNA según su sigla en inglés) [35, 40, 42]. El SNA se basa en modelar las diferentes interacciones entre individuos ya sea en equipos, organizaciones o cualquier otra forma de agrupación [43]. De esta manera, en primer lugar se siguió el procedimiento ocupado por Carson et al. [33] para medir la influencia entre cada par de estudiantes en un tiempo determinado. Así, cada estudiante respondió la siguiente pregunta sobre cada uno de sus compañeros del equipo “¿Este individuo aportó al liderazgo del equipo?”, teniendo que responder esta pregunta a la mitad y 
al final del trabajo. Esta pregunta fue respondida usando una escala binaria 0 o 1 , donde 1 se asocia a que el miembro del equipo aportó en el liderazgo del equipo y 0 en otro caso. En segundo lugar, para obtener el liderazgo total ejercido por cada individuo, se sumó la influencia que ejerció en cada uno de sus compañeros de equipo en la mitad y al final del trabajo para finalmente promediar estos valores. En tercer lugar, para obtener el valor de la densidad y centralización de la red, se siguieron los procedimientos realizados por investigaciones realizadas en liderazgo compartido que se basan en el análisis de redes sociales usando las ecuaciones 1 y $2[12,33,44]$. De esta manera, la densidad de la red se puede calcular a través de la fórmula 1 propuesta por Wasserman y Faust [45], donde en el numerador se presenta la suma de todas las relaciones de liderazgo desarrolladas en el equipo y este valor se divide por la cantidad máxima de relaciones de liderazgo que pueden haber en el equipo a través del denominador $\mathrm{n}(\mathrm{n}-1)$, donde $\mathrm{n}$ se refiere a la cantidad de individuos que hay en el equipo. Esta fórmula entrega valores que pertenecen al intervalo 0 a 1, donde valores más cercanos a 0 se refieren a redes menos densas y valores más cercanos a 1 se refieren a equipos donde la estructura de liderazgo es compartida por gran parte de los miembros [30].

$$
\text { Densidad }=\frac{\sum_{i=1}^{n} \text { Relaciones de liderazgo }_{i}}{n(n-1)}
$$

La centralización de la red puede entender como una medida de la variabilidad de las relaciones de liderazgo ejercidas por los estudiantes del equipo. Así, cuando en un equipo solo un estudiante desarrolla liderazgo, esta red está altamente centralizada, tomando valores cercanos a 1 mientras que en un equipo donde todos los individuos van ejerciendo el rol de líder, este indicador de la red se va acercando a 0 , siendo esta última una buena representación del liderazgo compartido. La ecuación 2 muestra como calcular el nivel de centralización de la red [45], donde $\mathrm{C}_{\mathrm{x}}\left(\mathrm{p}_{\mathrm{i}}\right)$ es grado de centralización individual que se refiere a la cantidad de influencia por el individuo i ejercida a los demás miembros del equipo. Así, primero se localiza el máximo valor que puede tener $\mathrm{C}_{\mathrm{x}}$ pensándolo como un individuo que es líder para todos los miembros del equipo. En segundo lugar, para cada miembro se calcula su propio valor de $\mathrm{C}_{\mathrm{x}} \mathrm{y}$ se le resta al valor máximo encontrado anteriormente para luego sumar todos estos valores. Por último, esta sumatoria se divide en el máximo de la sumatoria anteriormente señalada, el cálculo de este denominador puede ser más fácil de entender pensando a un equipo donde solo un individuo ejerce todo el liderazgo siendo el $\mathrm{C}_{\mathrm{x}}$ igual a 0 para todos los demás miembros del equipo [30].

$$
\text { Centralización }=\frac{\sum_{i=1}^{n}\left[\max C_{x}(p)-C_{x}\left(p_{i}\right)\right]}{\max \sum_{i=1}^{n}\left[\max C_{x}(p)-C_{x}\left(p_{i}\right)\right]}(2)
$$

Por último, para medir el desempeño de los equipos se utilizó la nota obtenida por cada equipo en el trabajo realizado.

\section{ANÁLISIS DE DATOS Y RESULTADOS}

La Tabla 1 muestra un resumen de los estadísticos descriptivos de la muestra para los 164 alumnos en 41 equipos.

Antes de realizar el análisis de datos, la Tabla 2 muestra las correlaciones entre las variables de estudio entregada por el software MPlus V8.5. Como se puede ver inicialmente, los datos recolectados señalan que la densidad de liderazgo se correlaciona de manera positiva con el desempeño mientras que la centralización del liderazgo se correlaciona de forma negativa.

Para poder determinar si las relaciones que se proponen tienen significancia estadística, se realizó un modelo de ecuaciones estructurales donde se plantean dos modelos simultáneos. El primero,

Tabla 1. Estadisticos descriptivos.

\begin{tabular}{|l|c|c|c|c|c|c|}
\cline { 2 - 7 } \multicolumn{1}{c|}{} & Hombres & Mujeres & Total de estudiantes & Edad Promedio & Desv. estándar edad & Equipos \\
\hline Estudio 1 & 38 & 50 & 88 & 20,966 & 1,418 & 22 \\
\hline Estudio 2 & 40 & 36 & 76 & 21,197 & 1,395 & 19 \\
\hline Muestra Total & 78 & 86 & 164 & 21,073 & 1,408 & 41 \\
\hline
\end{tabular}


Tabla 2. Correlaciones bivariadas.

\begin{tabular}{|l|c|c|c|c|c|}
\cline { 2 - 6 } \multicolumn{1}{c|}{} & Desempeño & Proporción de Mujeres & Edad Promedio & Densidad & Centralización \\
\hline Desempeño & 1 & & & & \\
\hline Proporción de Mujeres & 0,216 & 1 & & & \\
\hline Edad Promedio & 0,077 & 0,09 & 1 & & \\
\hline Densidad & $0,638^{* *}$ & 0,184 & $-0,093$ & 1 & \\
\hline Centralización & $-0,321^{*}$ & $-0,029$ & $-0,2$ & $-0,28^{*}$ & 1 \\
\hline
\end{tabular}

$* * \mathrm{p}<0,001$ у $\mathrm{p}<0,05$.

donde la densidad y las variables de control, como son la proporción de mujeres en el equipo y la edad promedio del equipo, se relacionan con el desempeño de los estudiantes y un segundo modelo que es idéntico al anterior, pero usando la centralización y no la densidad del liderazgo. La Tabla 3 muestra los resultados obtenidos en el modelo de ecuaciones estructurales entregado por el software MPlus V8.5.

Los resultados obtenidos muestran que, efectivamente, la densidad del liderazgo y la centralización del liderazgo de los estudiantes en los equipos pueden relacionarse con su desempeño. Esto quiere decir que cuando los estudiantes exhiben mayores niveles de liderazgo a nivel agregado, el desempeño del equipo es mejor que cuando hay un menor liderazgo agregado de los estudiantes $(\beta=0,634, \mathrm{p}<0,01)$. Además, cuando los equipos tienen un liderazgo más centralizado de uno o pocos estudiantes su desempeño es menor en comparación con equipos donde el liderazgo se distribuye en gran parte de los estudiantes $(\beta=-0,316, \mathrm{p}<0,05)$. Por último, la proporción de mujeres no muestra un efecto significativo en el desempeño en ninguno de los dos modelos, esto quiere decir que el efecto de la proporción de genero (ya sea hombre o mujeres) no genera diferencias significativas en el desempeño en los equipos. Estos resultados entregan soporte tanto a la Hipótesis 1 como a la Hipótesis 2 del estudio.

\section{CONCLUSIONES}

Esta investigación propone nuevas formas de entender el liderazgo entendiéndolo no como una característica individual sino como un proceso de equipo. Esta conceptualización ayuda a entender realmente el efecto que tiene este fenómeno en este caso en los estudiantes de educación superior ya que, aunque continuamente se hace referencia a la importancia del liderazgo en su proceso formativo, pocas veces este es medido y aún en menor medida relacionado con su desempeño. En este sentido, esta investigación entrega una serie de conclusiones que pueden dar herramientas para poder entender el liderazgo en los estudiantes.

En primer lugar, esta investigación encontró que el liderazgo en los equipos de estudiantes puede modelarse como una estructura de redes sociales a nivel del equipo. Debido a que se conceptualizó el liderazgo como un proceso de influencia entre estudiantes, donde además tenían el carácter de ser autogestionados, se pudieron establecer los nodos de la red y las relaciones en cada uno de los equipos. En segundo lugar, después de modelar la estructura de la red de liderazgo en cada equipo, se pudo relacionar de manera positiva la densidad de la red de liderazgo y de manera negativa la centralización de la red de liderazgo con el desempeño de los

Tabla 3. Resultados del Modelo de Ecuaciones Estructurales.

\begin{tabular}{|l|c|c|c|c|c|c|c|c|}
\cline { 2 - 9 } \multicolumn{1}{c|}{} & \multicolumn{4}{c|}{ Modelo 1 } & \multicolumn{4}{c|}{ Modelo 2 } \\
\cline { 2 - 10 } \multicolumn{1}{c|}{} & Estimador & S.E. & Est./S.E. & P-Value & Estimador & S.E. & Est./S.E. & P-Value \\
\hline Desempeño en Proporción de mujeres & 0,088 & 0,120 & 0,729 & 0,466 & 0,207 & 0,141 & 1,473 & 0,141 \\
\hline Edad Promedio & 0,128 & 0,119 & 1,081 & 0,280 & $-0,005$ & 0,148 & $-0,031$ & 0,975 \\
\hline Densidad & 0,634 & 0,120 & 5,271 & 0,000 & & & & \\
\hline Centralización & & & & & $-0,316$ & 0,137 & $-2,307$ & 0,021 \\
\hline
\end{tabular}


estudiantes. Estos resultados son consistentes con estudios anteriores y meta-análisis de liderazgo compartido [12, 31, 32, 34]. Estos resultados muestran que las instituciones de educación superior deben ser capaces de tener planes y estrategias para poder desarrollar el liderazgo en sus estudiantes ya que sus beneficios tanto en el desempeño académico como posteriormente en el mundo laboral están ampliamente correlacionadas [46, 47]. Así, aplicar el análisis de redes sociales como una herramienta de modelamiento y estadística puede contribuir al seguimiento del desarrollo del liderazgo en los estudiantes de educación superior.

Por último, este estudio no está ajeno de limitaciones. Podemos señalar que la muestra utilizada solamente incluye estudiantes de Ingeniería Civil Industrial. En este sentido, aunque no hubo una diversidad de planes de estudio, lo que se buscó fue asegurar el hecho de que el trabajo realizado cumpliese con las características para fomentar el liderazgo compartido como lo son las tareas interconectadas y las responsabilidades compartidas. Además, debido a que el trabajo tuvo una duración de un mes y medio aproximadamente, hubo efectos que probablemente no pudieron ser controlados en el desarrollo del liderazgo en el equipo. Este problema se buscó disminuir obteniendo dos medidas de liderazgo por estudiante con el fin de aumentar la variabilidad de los datos y así tener información más representativa de la muestra y del trabajo en equipo.

\section{AGRADECIMIENTOS}

Los autores agradecen a la Universidad Católica de la Santísima Concepción por el patrocinio entregado para esta investigación a través del proyecto DINREG 24-18.

\section{REFERENCIAS}

[1] R.G. Lord, D.V Day, S.J. Zaccaro, B. J. Avolio and A. H. Eagly. "Leadership in applied psychology: Three waves of theory and research". J. Appl. Psychol. Vol. $102 \mathrm{~N}^{\circ} 3$, pp. 434-451.2017. DOI: $10.1037 /$ apl0000089.

[2] R.G. Lord and K.J. Maher. "Perceptions of leadership and their implications in organizations". Applied Social Psychology and Organizational Settings. Vol. 6, pp. 129-154.1990.
[3] E. Rodríguez-Ponce, L. Pedraja-Rejas and F. Ganga-Contreras. "La relación entre los estilos de liderazgo y el desempeño de los equipos de dirección intermedia: Un estudio exploratorio desde Chile". Contab. y Negocios. Vol. 12 Nº 23, pp. 129-144. 2017. DOI: 10.18800/contabilidad. 201701.009.

[4] L. Pedraja-Rejas, R.V. Massó and J.R. Castañeda. "La importancia de los estilos de liderazgo en la calidad de las unidades académicas universitarias". Opción Rev. Ciencias Humanas y Soc. Vol. $34 \mathrm{~N}^{\circ} 86$, pp. 130-151. 2018.

[5] V. Arévalo Almirón and A. Tikhomirova. "Liderazgo transaccional vs Liderazgo transformacional". Reidocrea. Vol. 4, pp. 24-27. 2015.

[6] R. Mahsud, G. Yukl and G.E. Prussia. "Leader empathy, ethical leadership, and relations-oriented behaviors as antecedents of leader-member exchange quality". J. Manag. Psychol. Vol. 25 No 6, pp. 561-577. 2010. DOI: $10.1108 / 02683941011056932$.

[7] B. Schyns. "Leader and follower personality and LMX". The Oxford Handbook of LeaderMember Exchange. $\mathrm{N}^{\mathrm{o}}$ June. 2015.

[8] K.L. Cullen-Lester and F.J. Yammarino. "Collective and network approaches to leadership: Special issue introduction". Leadersh Q. Vol. 27 N² 2, pp. 173-180. 2016. DOI: 10.1016/j.leaqua. 2016.02.001.

[9] R.C. Barnett and N.K. Weidenfeller. "Shared Leadership and Team Performance". Adv. Dev. Hum. Resour. Vol. 18 N $^{\circ} 3$, pp. 334-351. 2016. DOI: $10.1177 / 1523422316645885$.

[10] C.L. Pearce and H.P. Sims. "Shared leadership: Toward a multi-level theory of leadership". Adv. Interdiscip. Stud. Work Teams. Vol. 7, pp. 115-139. 2000.

[11] F.J. Yammarino, E. Salas, A. Serban, K. Shirreffs and M.L. Shuffler. "Collectivistic Leadership Approaches: Putting the 'We' in Leadership Science and Practice". Ind. Organ. Psychol. Vol. $5 \mathrm{~N}^{\circ}$ 4, pp. 382-402. 2012. DOI: $10.1111 /$ j.1754-9434.2012.01467.x.

[12] D. S. DeRue, J. D. Nahrgang and S. J. Ashford. "Interpersonal perceptions and the emergence of leadership structures in groups: A network perspective". Organ. Sci. Vol. 26 $\mathrm{N}^{\circ}$ 4, pp. 1192-1209. 2015. DOI: $10.1287 /$ orsc.2014.0963. 
[13] T. L. Friedrich, W.B. Vessey, M.J. Schuelke, G.A. Ruark and M.D. Mumford. "A framework for understanding collective leadership: The selective utilization of leader and team expertise within networks". United States Army Research Institute for the Behavioral and Social Sciences. Vol. 20 $\mathrm{N}^{\circ}$ 6. 2009.

[14] O. Epitropaki, R. Kark, C. Mainemelis and R.G. Lord. "Leadership and followership identity processes: A multilevel review". Leadersh Q. Vol. $28 \mathrm{~N}^{\circ}$ 1, pp. 104-129. 2017. DOI: 10.1016/j. leaqua. 2016.10.003.

[15] D.R. Carter, L.A. DeChurch, M.T. Braun and N.S. Contractor. "Social network approaches to leadership: An integrative conceptual review". J. Appl. Psychol. Vol. $100 \mathrm{~N}^{\circ} 3$, pp. 597-622. 2015. DOI: 10.1037/a0038922.

[16] C. Emery, T.S. Calvard and M.E. Pierce. "Leadership as an emergent group process: A social network study of personality and leadership". Gr. Process. Intergr. Relations. Vol. $16 \mathrm{~N}^{\circ}$ 1, pp. 28-45. 2013. DOI: $10.1177 / 1368430212461835$.

[17] J.F. Molina-Azorín, J. Pereira-Moliner, M.D. López-Gamero, E.M. Pertusa-Ortega and J.J. Tarí. "Multilevel research: Foundations and opportunities in management". BRQ Bus. Res. Q. 2019.

[18] V. Ekimova and A. Kokurin. "Students" attitudes towards different team building methods". Procedia - Soc. Behav. Sci. Vol. 186, pp. 847-855. 2015. DOI: 10.1016/j. sbspro.2015. 04.157.

[19] J.A. Pareja Fernández de la Reguera, J.A. López Núñez, M. El Homrani y R. Lorenzo Martín. "El liderazgo en los estudiantes universitarios: Una fructífera línea de investigación”. Educar. Vol. 48 Nº 1, pp. 91 119. 2012.

[20] P. Robledo, R. Fidalgo, O. Arias y M.L. Álvarez."Percepción de los estudiantes sobre el desarrollo de competencias a través de diferentes metodologías activas". Rev. Investig. Educ. Vol. $33 \mathrm{~N}^{\circ}$ 2, pp. 369-383. 2015. DOI: 10.6018/rie.33.2.201381.

[21] A.A. Aymoldanovna, B.A. Zhetpisbaeva, K.U. Kozybaevna and S.M. Kadirovna. "Leadership development university students in the activities of student government". Procedia Soc. Behav. Sci. Vol. 197 N ${ }^{\circ}$ February, pp. 2131-2136. 2015. DOI: 10.1016/j.sbspro. 2015.07.336.

[22] E. Rodríguez-Ponce. "El rol de las universidades en la sociedad del conocimiento y en la era de la globalización: Evidencia desde Chile". Interciencia. Vol. $34 \mathrm{~N}^{\circ} 11$, pp. 822-829. 2009.

[23] R. Bolden, S. Jones, H. Davis and P. Gentle. "Developing and sustaining shared leadership in higher education". Leadership Foundation for Higher Education. 2015.

[24] G. Coluccio. "Entendiendo el rol de la personalidad en el surgimiento del liderazgo compartido en sus diferentes niveles de análisis”. Universidad Adolfo Ibañez. 2018.

[25] J. Lumby. "Leadership and power in higher education". Stud. High. Educ. Vol. Issue 9, pp. 1619-1629.2018. DOI: 10.1080/03075079.2018.1458221.

[26] M. López Gomez, M.P. Cáceres Reche and M. Agreda Montoro. "Estudio sobre liderazgo estudiantil desde un enfoque socio-político en la universidad Santo Tomás de Bucaramanga (Colombia)". Int. J. Educ. Leadersh. Manag. Vol. $5 \mathrm{~N}^{\circ}$ 2, pp. 178. 2017. DOI: $10.17583 /$ ijelm. 2017. 2514.

[27] M. Lorenzo Delgado. "El liderazgo en las organizaciones educativas: Revisión y perspectivas actuales". Rev. española Pedagog. Vol. 63 N$^{\circ}$ 232, pp. 367-388. 2005.

[28] L. Pedraja-Rejas y J. Riquelme-Castañeda. "El estudio de los estilos de liderazgo en el campo de la educación". Interciencia. Vol. 42 No 11, pp. 709-709. 2017.

[29] M.L. Delgado. "Las comunidades de liderazgo de centros educativos". 2012.

[30] C. Gockel and L. Werth. "Measuring and modeling shared leadership: Traditional approaches and new ideas". J. Pers. Psychol. Vol. $9 \mathrm{~N}^{\circ}$ 4, pp. 172-180. 2011. DOI: 10.1027/1866-5888/a000023.

[31] L. D'Innocenzo, J.E. Mathieu and M.R. Kukenberger. "A Meta-Analysis of Different Forms of Shared LeadershipTeam Performance Relations". J. Manage. Vol. $42 \mathrm{~N}^{\circ}$ 7, pp. 1964-1991. 2016. DOI: $10.1177 / 0149206314525205$.

[32] D. Wang, D.A. Waldman and Z. Zhang. "A meta-analysis of shared leadership and team effectiveness". J. Appl. Psychol. Vol. 99 N $^{\circ} 2$, pp. 181-198. 2014. DOI: 10.1037/a0034531. 
[33] J.B. Carson, P.E. Tesluk and J.A. Marrone. "Shared leadership in teams: An investigation of antecedent conditions and performance". Acad. Manag. J. Vol. 50 N 5, pp. 1217-1234. 2007. DOI: $10.2307 / 20159921$.

[34] V.C. Nicolaides, K.A. LaPortTiffani, R.Chen, A.J.Tomassetti, E.J.Weis, S.J. Zaccaro and Jose M. Cortina."The shared leadership of teams: A meta-analysis of proximal, distal, and moderating relationships". Leadersh Q. Vol. $25 \mathrm{~N}^{\circ}$ 5, pp. 923-942. 2014. DOI: 10.1016/j.leaqua. 2014.06.006.

[35] D.S. DeRue. "Adaptive leadership theory: Leading and following as a complex adaptive process". Res. Organ. Behav. Vol. $31 \mathrm{~N}^{\circ} 2$, pp. 125-150. 2011. DOI: 10.1016/j.riob. 2011.09.007.

[36] C.L. Pearce and J.A. Conger. "Shared leadership: Reframing the hows and whys of leadership". SAGE Publications. 2003.

[37] G. Coluccio, L. Pedraja-Rejas, C. Medel Romero y N. Meza Castro."Estilo de liderazgo pasivo-evitador e intercambio de comportamientos líder-seguidor en estudiantes universitarios: Una aproximación desde Chile". Hallazgos. Vol. 18 No 35.2021. DOI: $10.15332 / 2422409 x .5848$.

[38] E.E. Small and J.R. Rentsch. "Shared Leadership in Teams". J. Pers. Psychol. Vol. $9 \mathrm{~N}^{\circ} 4$, pp. 203-211. 2010. DOI: 10.1027/1866-5888/a000017.

[39] B.A. De Jong, K.T. Dirks and N. Gillespie. "Trust and team performance: A metaanalyis of main effects, moderators and covariates". J. Appl. Psychol. Vol. 101 No 8, pp. 1134-1150. 2015. DOI: 10.1017/ CBO9781107415324.004.
[40] M. Mccusker. "A Dyadic Approach to Leadership Emergence". Virginia Polytechnic Institute and State University. 2015.

[41] N.S. Contractor, L.A. DeChurch, J.B. Carson, D.R. Carter and B. Keegan. "The topology of collective leadership". Leadersh Q. Vol. 23 $\mathrm{N}^{\circ}$ 6, pp. 994-1011. 2012. DOI: 10.1016/j. leaqua. 2012. 10.010.

[42] E. Adriasola and R.G. Lord. "From a leader and a follower to shared leadership: An identity-based structural model for shared leadership emergence". The Connecting Leader: Serving Concurrently as Leader and a Follower. Information Age Publishing. 2020.

[43] A.J. O'Malley and P.V. Marsden. "The analysis of social networks". Heal. Serv. Outcomes Res. Methodol. Vol. $8 \mathrm{~N}^{\mathrm{o}}$ 4, pp. 222-269. 2008. DOI: 10.1007/s10742-008-0041-z.

[44] M. Mayo, J.R. Meindl and J.C. Pastor. "Shared leadership in work teams: A social network approach". Sage Books, pp. 193214. 2003.

[45] S. Wasserman and K. Faust. "Social network analysis: Methods and applications". 1st edition. Cambridge University Press. Vol. 8. Cambridge, UK. 1994.ISBN: 9780511815478.

[46] K. Kearns. "The Leadership Portfolio Program at The University of Pittsburgh: Teaching leadership to graduate students". Teach. Public Adm. Vol. 37 No 3, pp. 255-273. 2019. DOI: 10. 1177/0144739419851143.

[47] A.M. Corriveau. "Developing authentic leadership as a starting point to responsible management: A Canadian university case study". Int. J. Manag. Educ. Vol. $18 \mathrm{~N}^{\mathrm{o}} 1$. 2020. DOI: 10.1016/j.ijme. 2020.100364. 